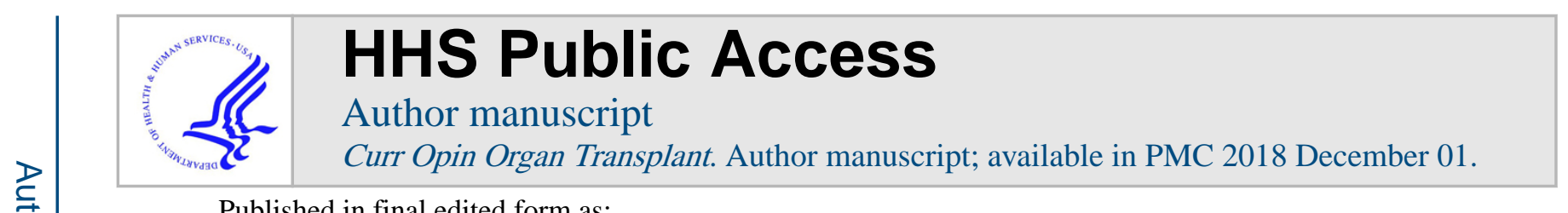

Published in final edited form as:

Curr Opin Organ Transplant. 2017 December ; 22(6): 535-540. doi:10.1097/MOT.0000000000000459.

\title{
Liver Xenotransplantation
}

\author{
Madhukar S. Patel, MD, MBA, ScM ${ }^{1,2}$, Nathan Louras, MD¹, and Parsia A. Vagefi, MD, \\ FACS $^{1,2,3}$ \\ ${ }^{1}$ Department of Surgery, Massachusetts General Hospital, Boston, Massachusetts \\ ${ }^{2}$ Harvard Medical School, Boston, Massachusetts
}

\begin{abstract}
Purpose of Review-There continues to be inadequate organ supply, and lack of effective temporary support, for patients with liver failure. The purpose of this review is to discuss recent progress in the field of orthotopic pig-to-nonhuman primate (NHP) liver xenotransplantation (LXT).
\end{abstract}

Recent Findings-From 1968 to 2012, survival in pig-to-NHP LXT has been limited to 9 days, initially due to hyperacute rejection which has been ameliorated through use of genetically engineered donor organs, but ultimately due to profound thrombocytopenia, thrombotic microangiopathy (TMA), and bleeding. More recently, demise secondary to lethal coagulopathy was avoided with LXT of a (1,3)-galactosyltransferase (GT) knockout (GTKO), CMV-negative porcine xenografts into baboons receiving exogenous administration of coagulation factors and costimulation blockade, establishing that a porcine liver is capable of supporting NHP life for nearly a month.

Summary-Continued consistent achievement of pig-to-NHP LXT survival beyond two weeks justifies consideration of a clinical application as a bridge to allotransplantation for patients with acute hepatic failure. Further genetic modifications to the donor, as well as additional studies, are required in order to apply LXT as destination therapy.

\section{Keywords}

Liver; Pig; Nonhuman Primate; Xenotransplantation; Bridge

\section{INTRODUCTION}

The growing success of liver transplantation over the past five decades has led to a demand for organs that far outpaces supply. Most recently in 2016, there were 7,810 liver transplants performed in the United States. During this time, however, 1,431 patients were removed from the waiting list as they were felt to be too sick to transplant, and an additional 1,233 patients died while awaiting liver transplantation (based on OPTN data as of May 2017).

\footnotetext{
${ }^{3}$ Parsia A. Vagefi, M.D., Massachusetts General Hospital, 55 Fruit Street, White 521c, Boston, MA 02114, Phone 617-726-8606, Fax 617-726-8137, pvagefi@mgh.harvard.edu.

CONFLICTS OF INTEREST

None.
} 
The demise of the patient with liver failure is perhaps most tragic in the population of candidates suffering from acute liver failure (ALF) which often occurs in those without preexisting liver disease.(1) Indeed, prior to the introduction of liver transplantation, these patients had an $80 \%$ mortality rate with up to $40 \%$ of adult ALF patients dying during their index hospitalization.(2) ALF patients who die awaiting transplant spend an average of three days on the transplant waitlist prior to their death, highlighting that it is the critical shortage of organs which facilitates their demise. $(3,4)$ The two-thirds of ALF candidates who are able to achieve emergent liver transplantation, however, have reported 2-year survival of $92 \% .(2)$

In addition to a shortage in supply of liver grafts available for transplantation, the lack of an effective means of providing temporary liver support to help sustain hepatic function and treat progression of accompanying medical disease, has compounded the need to develop solutions that ultimately enable liver transplantation. Specifically, although the United States (US) Food and Drug Administration (FDA) has approved artificial liver support systems (ALS) such as the molecular adsorbent recirculating system (MARS) for use in patients with acute toxic liver injury and refractory encephalopathy, an overall survival advantage has not been clearly demonstrated and wide adoption of the technology has been limited $(5,6)$.

To address the issue of organ supply and resultant demise of patients with liver failure, clinical application of porcine xenogeneic livers has remained an attractive goal, initially as a bridge to allotransplantation and, eventually, as destination therapy. There are numerous reasons that pigs would be an ideal source for liver xenotransplantation (LXT), especially when compared to other potential donors such as non-human primates (NHPs).(7) Namely, they have good breeding potential, rapid growth, adequate adult organ size, relatively low cost of maintenance, and close anatomic and physiology similarity to humans.(7) Additionally, considerable knowledge and experience has been gained over the years with regards to tissue typing and genetic engineering making their potential to be optimal donors more likely.(7) Until recently, challenges related to the consistent development of lethal coagulopathy has troubled progress in the field of pig-to-NHP LXT, however, reports suggesting prolonged survival of nearly a month have reinvigorated interest (FIGURE 1). (8-10) In this review, progress in the field of orthotopic pig-to-NHP LXT will be discussed as it pertains to laboratory developments in large animal models that have informed clinical trials which are under consideration.

\section{LABORATORY EXPERIENCE WITH LIVER XENOTRANSPLANTATION}

\section{Wild-type porcine donors}

The promise of LXT has persisted since initial observations made in large animal experiments performed in 1968.(11) In this pioneering study by Calne et al., seven pig-tobaboon orthotopic liver xenotransplants were performed with survival up to 3.5 days.(11) The longest surviving animal in this initial series received steroids and azathioprine for immunosuppression and died from bronchopneumonia with necropsy notable for preserved hepatocytes and evidence of mononuclear cell infiltration of the portal tracts.(11) Subsequent attempts two years later using rhesus monkey and chimpanzee as recipients were less successful and notable for recipient death in less than 12 hours with evidence of diffuse 
intravascular coagulation.(12) The following two decades would see additional attempts at LXT utilizing wild-type (WT) pigs as donors, but with continued limited survival of less than three days. $(13,14)$

\section{Genetically-engineered porcine donors}

The consistent development of hyperacute rejection within hours and days, suggested that interspecies incompatibilities might preclude even short-term clinical application of LXT, and resulted in a scientific trajectory aimed at donor modification. $(15,16)$ Applying developments in genetic engineering, the introduction of genes in donor pigs for complement-regulatory proteins or the deletion of specific genes responsible for producing antigens for which primates have natural antibodies has been performed. In 2000, Ramirez et al. performed the first pig-to-NHP LXT using a genetically engineered pig expressing human complement regulatory decay accelerating factor (h-DAF or CD55).(17) In this study, baboons with livers from h-DAF transgenic pigs survived for up to 8 days, without evidence of hyperacute rejection due to the activation of recipient complement in the graft. (17) Clotting factors were found to be synthesized by the h-DAF xenografts and reached near normal levels and had evidence of in vivo function.(17) In this series, the animal with the longest survival ultimately needed to be euthanized due to the development of septicemia that presumably led to disseminated intravascular coagulopathy.(17)

With encouraging results of transgenic pig liver xenografts expressing h-DAF, Ramierez et al. developed polytransgenic pig livers for transplant.(18) These polygenic pigs expressed hDAF in addition to the human complement regulator protein CD59 and alfa 1,2fucosyltransferase (H-transferase).(18) Although recipients of polygenic liver xenografts were noted to have absence of hyperacute rejection and preserved liver architecture on necropsy, survival was limited to between 13 and 24 hours with cause of death being thrombosis in three cases and primary xenograft dysfunction in one case.(18)

In addition to donor modifications that introduce new genes, transplantation of livers with deletion of genes which are felt to lead to interspecies incompatibilities has been also been successfully accomplished. Specifically, the finding that all mammals below old world monkeys (e.g. pigs) express the Gal epitope, and those equivalent and above (e.g humans) have natural, or preformed, anti-Gal antibodies that target this epitope motivated efforts to produce $\mathrm{a}(1,3)$-galactosyltransferase (GT) knockouts (GTKO).(19, 20) Ekser et al. reported the first results of LXT with GTKO pigs as well as those polytransgenic for GTKO and CD46. $(15,21)$ Clinically, this study suggested that survival from 4-7 days could be achieved and during this time, the liver xenograft was capable of adequate protein synthesis (including albumin, fibrinogen, haptoglobin, plasminogen, and coagulation factors).(15, 21) Unfortunately, however, recipients developed profound thrombocytopenia which began to develop within an hour after reperfusion and eventually resulted in spontaneous hemorrhage which did not allow for survival beyond one week.(21) In a subsequent study of GTKO liver xenografts preformed by Kim et al., utilizing a different immunosuppression regimen (FIGURE 1), survival of 6, 8, and 9 days was accomplished.(16) In the two longest survivors, aminocaproic acid (Amicar) was administered. As a plasmin inhibitor, Amicar inhibited fibrinolysis and it was felt that this helped maintain platelet counts above 40,000 
per $\mathrm{mm}^{3}$. Unrelated to thrombocytopenia, however, these recipients had persistent blood loss and hemolysis suggestive of ongoing coagulopathy and sepsis which were ultimately lethal. Notably, at the time of necropsy there was neither evidence of acute cellular nor humoral rejection.

\section{Exogenous coagulation factor administration}

Despite the development of h-Daf transgenic and GTKO pigs over a decade ago(22), until recently, maximum liver xenograft survival for the last 50 years had been limited to 9 days. (16) As mentioned, the demise of the primate liver xenograft recipient has been one characterized by dysregulated coagulation which manifests as a profound thrombocytopenia, bleeding, and thrombotic microangiopathy (TMA) $(15,16)$, presumed to be due to the activation of the porcine vascular endothelium by low levels of anti-nonGal antibody. Furthermore, historical observations have demonstrated that naive pigs and baboons possess inherent differences in baseline coagulation factor activity. This was most notable in the absence of (or inability to detect with human coagulation factor detection assays) circulating Factor VII(a) in naive pigs.(16) Following LXT, Factor VII activity was only detected on POD 0 and likely represented residual circulating baboon Factor VII, as by POD 2 porcine Factor VII levels had returned to being undetectable in LXT recipients.(16) Given these findings, an attempt to address the coagulation dysregulation using exogenous human coagulation factor administration six hours following pig-to-baboon LXT was performed.(8) Two different pharmacological agents: Octaplex ${ }^{\circledR}$, a second-generation human prothrombin complex concentrate (hPCC) containing the factors II, VII, IX, and X, as well as Proteins C and S, and NovoSeven ${ }^{\circledR}$, human recombinant activated Factor VII, were used.(8) hPCC was administered in both bolus and continuous fashion, whereas Factor VIIa was given only as a continuous infusion.(8)

Bolus administration of hPCC resulted in significant large and small vessel graft thrombosis with survival of only 1 and 3 days in 2 recipients. In contrast, recipients of continuous coagulation factor administration $(n=4)$ experienced a substantially decreased transfusion requirement as well as an absence of histological evidence of TMA within the xenografts.(8) Indeed, continuous infusion LXT recipients required only approximately $200 \mathrm{cc}$ of blood to maintain a week's long survival. In contrast, historical controls performed without coagulation factor infusions required approximately $600 \mathrm{cc}$ of blood over the same time frame. Interestingly, the continuous administration of human coagulation factors resulted in either stabilization or an increase in the recipient circulating platelet levels.(8) Despite the avoidance of TMA within the graft and the appearance of spontaneous peripheral platelet count recovery, survival continued to be limited to less than 7 days.

\section{Co-stimulation blockade}

On the basis of the aforementioned findings, and with recent reports of improved kidney and heart xenotransplant survival utilizing co-stimulation blockade(23, 24), Shah et. al developed a modified protocol which integrated the administration of exogenous coagulation factors with co-stimulation blockade for baboon recipients of pCMV-free GTKO porcine donors (FIGURE 2). With these modifications, four additional pig-to-baboon LXT experiments were performed using a continuous infusion of hPCC and immunosuppression consisting of 
anti-thymocyte globulin induction and maintenance therapy with FK-506, methylprednisone and either belatacept $(n=3)$ or anti-CD40 monoclonal antibody $(m A b)(n=1) .(9,10)$ All four recipients demonstrated a significant reduction in blood transfusion requirements postoperatively and spontaneous recovery of platelet counts beginning as early as POD 4, with peak platelet counts of 614,000 and 709,000 in the POD 25 and POD 29 surviving animals, respectively.(10) This degree of platelet count recovery was likely associated with the postsplenectomy state, with subsequent normalization of this reactive thrombocytosis in the weeks following splenectomy.

Thus, a novel regimen consisting of continuous exogenous human coagulation factor and costimulation blockade in baboon recipients of porcine CMV negative xenografts, has allowed not only for the previously lethal coagulopathy to be circumvented, but also has achieved for the first time nearly one-month survival following pig-to-baboon LXT. $(8,9)$ This emphasizes that a NHP can be maintained with the hepatic metabolism of a porcine liver, and holds promise at this early stage for consideration of initial clinical application of LXT as a bridge to allotransplantation in patients with acute hepatic failure.

\section{CLINICAL EXPERIENCE WITH LIVER XENOTRANSPLANTATION}

\section{Orthotopic transplantation}

To date, the only case of hepatic xenotransplantation from a pig donor to a human was performed by Makowka et al. in a 26 year old female patient with fulminant hepatitis and marked encephalopathy and coagulopathy.(3) Due to the worsening condition of the recipient and lack of human donor organ availability, a decision was made to attempt to use a pig liver. The donor graft was obtained from mature, wild type, 20-kg Yucatan miniature pigs. In order to conduct preoperative plasmapheresis and remove recipient natural anti-pig antibodies so as to decrease the potential for antibody-mediated rejection, the donor kidneys were perfused ex-vivo. Immunosupression included azathioprine, cyclophosphamide, cyclosporine, and methylprednisolone. After one hour of plasmapheresis, the pig liver xenograft was heterotopically transplanted inferior to the patient's native liver. A heterotropic approach was chosen due to the decline in the patient's condition and risk associated with donor hepatectomy.

Postoperatively, improvement was noted in all organ systems except the central nervous system. Specifically, liver xenograft function was noted by initial bile production, improved lactate clearance, stabilization of prothrombin times, decreased serum bilirubin levels, and decreasing transaminases. Due to worsening neurologic symptoms at 24 hours postoperatively, the patient was taken to the operating room as a human donor graft had become available. At this point the pig xenograft was removed and was noted to be enlarged and congested. Given progressive neurologic failure, however, the allotransplant effort was aborted and the patient was ultimately declared brain dead.

On immunologic studies, despite successful plasmapheresis of anti-pig antibodies prior to xenotransplant, antibody measurement after the LXT demonstrated a rise in anti-donor antibody within hours. Histologically, there was evidence of vascular damage at 165 minutes post-transplantation. At necropsy, extensive hemorrhagic necrosis and numerous occlusive 
thromboses of multiple different vessels including the hepatic arteries, central veins, and portal veins was noted. The cause of death in the patient was noted to be from cerebellar herniation secondary to cerebral edema.

\section{Clinical Trial Consideration}

With prolonged survival of orthotopic LXT using genetically-engineered porcine livers, clinical trial consideration is underway for use of this donor supply as a bridge in human transplantation. Appropriate patient selection is paramount for planning safe, ethical, and informative studies. As such, initial trials should focus on including those patients who are unable to wait and in whom no alternative therapy is available.(25) Clinically, these patients often have the one of the follow three clinical conditions: 1) ALF, 2) primary liver allograft failure due to primary non function or hepatic artery thrombosis within seven days of transplant, or 3) acute decompensation in the setting of chronic liver disease.(25) In these individuals who would otherwise die due to lack of organ availability, experimental application of LXT could serve as a bridge until an organ is available for allotransplantation. (25) Assuming success with these initial clinical trials assessing the safety and efficacy of orthotopic LXT, destination therapy can then ultimately be pursued.

\section{CONCLUSIONS}

Advances in donor management, surgical technique, and post-operative immunosuppression over the past five decades has led to excellent survival in liver transplant recipients. Despite this, however, the critical challenge remains a shortage of organs, which when accentuated by the absence of effective temporary liver support, leads to progression of disease and waitlist mortality. Encouragingly, recent progress in pig-to-NHP LXT has led to recipient survival of nearly a month. Indeed, with continued achievement of greater than two weeks' survival in this model, clinical translation as a bridge to liver allotransplantation could be enabled, most notably for the population of patients with ALF in dire need of immediate liver transplantation. With translational impact clearly in sight, it is imperative that continued work in the field of LXT remain a research priority.

\section{Acknowledgments}

\section{FINANCIAL SUPPORT AND SPONSORSHIP}

Nathan Louras was supported by an NIAID 5T32AI007529-18 training grant.

\section{References}

1. Bernal W, Wendon J. Acute liver failure. N Engl J Med. 2013; 369(26):2525-2534. [PubMed: 24369077]

2. Fontana RJ, Ellerbe C, Durkalski VE, Rangnekar A, Reddy RK, Stravitz T, et al. Two-year outcomes in initial survivors with acute liver failure: results from a prospective, multicentre study. Liver Int. 2015; 35(2):370-380. [PubMed: 25039930]

3. Makowa L, Cramer DV, Hoffman A, Breda M, Sher L, Eiras-Hreha G, et al. The use of a pig liver xenograft for temporary support of a patient with fulminant hepatic failure. Transplantation. 1995; 59(12):1654-1659. [PubMed: 7604434]

4. Lee WM, Squires RH Jr, Nyberg SL, Doo E, Hoofnagle JH. Acute liver failure: Summary of a workshop. Hepatology. 2008; 47(4):1401-1415. [PubMed: 18318440] 
5. Gerth HU, Pohlen M, Tholking G, Pavenstadt H, Brand M, Wilms C, et al. Molecular adsorbent recirculating system (MARS) in acute liver injury and graft dysfunction: Results from a case-control study. PLoS One. 2017; 12(4):e0175529. [PubMed: 28403210]

6. Banares R, Nevens F, Larsen FS, Jalan R, Albillos A, Dollinger M, et al. Extracorporeal albumin dialysis with the molecular adsorbent recirculating system in acute-on-chronic liver failure: the RELIEF trial. Hepatology. 2013; 57(3):1153-1162. [PubMed: 23213075]

7. Cooper DK, Bottino R. Recent advances in understanding xenotransplantation: implications for the clinic. Expert Rev Clin Immunol. 2015; 11(12):1379-1390. [PubMed: 26548357]

8**. Navarro-Alvarez N, Shah JA, Zhu A, Ligocka J, Yeh H, Elias N, et al. The Effects of Exogenous Administration of Human Coagulation Factors Following Pig-to-Baboon Liver Xenotransplantation. Am J Transplant. 2016; 16(6):1715-1725. Original research deomonstrating the beneficial impact of exogenous coagulation factor administration on transfusion requirements, platelets counts, and hepatic thrombotic microangiopathy. [PubMed: 26613235]

9. Shah JA, Navarro-Alvarez N, DeFazio M, Rosales IA, Elias N, Yeh H, et al. A Bridge to Somewhere: 25-day Survival After Pig-to-Baboon Liver Xenotransplantation. Ann Surg. 2016; 263(6):1069-1071. [PubMed: 26825261]

10**. Shah JA, Patel MS, Elias N, Navarro-Alvarez N, Rosales I, Wilkinson RA, et al. Prolonged Survival Following Pig-to-Primate Liver Xenotransplantation Utilizing Exogenous Coagulation Factors and Costimulation Blockade. Am J Transplant. 2017 Report detailing protocol used to achieve the longest surgival following pig-to-primate liver xenotransplant to date.

11. Calne RY, White HJ, Herbertson BM, Millard PR, Davis DR, Salaman JR, et al. Pig-to-baboon liver xenografts. Lancet. 1968; 1(7553):1176-1178. [PubMed: 4172293]

12. Calne RY, Davis DR, Pena JR, Balner H, de Vries M, Herbertson BM, et al. Hepatic allografts and xenografts in primates. Lancet. 1970; 1(7638):103-106.

13. Powelson J, Cosimi AB, Austen W Jr, Bailen M, Colvin R, Gianello P, et al. Porcine-to-primate orthotopic liver transplantation. Transplant Proc. 1994; 26(3):1353-1354. [PubMed: 8029937]

14. Luo Y, Kosanke S, Mieles L, Kobayashi T, Li SF, Niekrasz M, et al. Comparative histopathology of hepatic allografts and xenografts in the nonhuman primate. Xenotransplantation. 1998; 5(3):197206. [PubMed: 9741458]

15. Ekser B, Echeverri GJ, Hassett AC, Yazer MH, Long C, Meyer M, et al. Hepatic function after genetically engineered pig liver transplantation in baboons. Transplantation. 2010; 90(5):483-493. [PubMed: 20606605]

16. Kim K, Schuetz C, Elias N, Veillette GR, Wamala I, Varma M, et al. Up to 9-day survival and control of thrombocytopenia following alpha1,3-galactosyl transferase knockout swine liver xenotransplantation in baboons. Xenotransplantation. 2012; 19(4):256-264. [PubMed: 22909139]

17. Ramirez P, Chavez R, Majado M, Munitiz V, Munoz A, Hernandez Q, et al. Life-supporting human complement regulator decay accelerating factor transgenic pig liver xenograft maintains the metabolic function and coagulation in the nonhuman primate for up to 8 days. Transplantation. 2000; 70(7):989-998. [PubMed: 11045632]

18. Ramirez P, Montoya MJ, Rios A, Garcia Palenciano C, Majado M, Chavez R, et al. Prevention of hyperacute rejection in a model of orthotopic liver xenotransplantation from pig to baboon using polytransgenic pig livers (CD55, CD59, and H-transferase). Transplant Proc. 2005; 37(9):41034106. [PubMed: 16386637]

19. Galili U, Rachmilewitz EA, Peleg A, Flechner I. A unique natural human IgG antibody with antialpha-galactosyl specificity. J Exp Med. 1984; 160(5):1519-1531. [PubMed: 6491603]

20. Galili U, Shohet SB, Kobrin E, Stults CL, Macher BA. Man, apes, and Old World monkeys differ from other mammals in the expression of alpha-galactosyl epitopes on nucleated cells. J Biol Chem. 1988; 263(33):17755-17762. [PubMed: 2460463]

21. Ekser B, Long C, Echeverri GJ, Hara H, Ezzelarab M, Lin CC, et al. Impact of thrombocytopenia on survival of baboons with genetically modified pig liver transplants: clinical relevance. Am J Transplant. 2010; 10(2):273-285. [PubMed: 20041862]

22. Kolber-Simonds D, Lai L, Watt SR, Denaro M, Arn S, Augenstein ML, et al. Production of alpha-1,3-galactosyltransferase null pigs by means of nuclear transfer with fibroblasts bearing loss 
of heterozygosity mutations. Proc Natl Acad Sci U S A. 2004; 101(19):7335-7340. [PubMed: 15123792]

23**. Mohiuddin MM, Singh AK, Corcoran PC, Thomas ML 3rd, Clark T, Lewis BG, et al. Chimeric 2C10R4 anti-CD40 antibody therapy is critical for long-term survival of GTKO.hCD46. hTBM pig-to-primate cardiac xenograft. Nat Commun. 2016; 7:11138. Study demonstrating the positive impact of co-stimulation blockade on survival after heart xenotransplantation. [PubMed: 27045379]

24. Higginbotham L, Mathews D, Breeden CA, Song M, Farris AB 3rd, Larsen CP, et al. Pretransplant antibody screening and anti-CD154 costimulation blockade promote long-term xenograft survival in a pig-to-primate kidney transplant model. Xenotransplantation. 2015; 22(3): 221-230. [PubMed: 25847130]

25*. Cooper DK, Wijkstrom M, Hariharan S, Chan JL, Singh A, Horvath K, et al. Selection of Patients for Initial Clinical Trials of Solid Organ Xenotransplantation. Transplantation. 2016Review article detailing considerations for clinical trails in xenotransplantion 


\section{KEY BULLET POINTS}

- Until recently, advances in orthotropic pig-to-nonhuman primate (NHP) liver xenotransplantation (LXT) have been limited by lethal coagulopathy manifesting as profound thrombocytopenia, bleeding, and thrombotic microangiopathy (TMA).

- Through use of a (1,3)-galactosyltransferase (GT) knockout (GTKO), CMVnegative porcine xenografts, baboons receiving exogenous administration of coagulation factors and co-stimulation blockade are capable of surviving for nearly a month.

- In individuals who would otherwise die due to lack of organ availability, experimental application of LXT has potential to serve as a bridge until an organ is available for allotransplantation. 


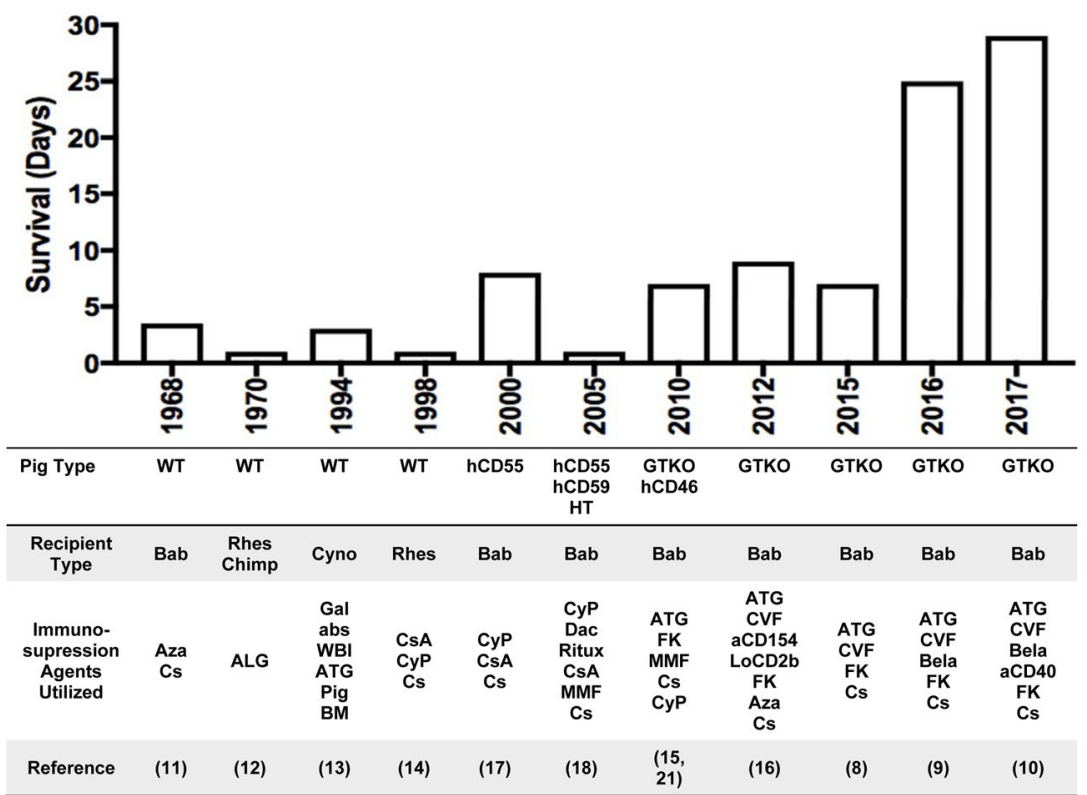

Figure 1.

Progress in survival of pig-to-non human primate orthotopic xenoliver transplantation. aCD40 = anti-CD40 monoclonoal antibodies; aCD154, anti-CD154 monoclonal antibodies; ALG, antilymphocyte globulin; ATG, antithymocyte globulin; Aza, azathioprine; Bab, baboon; Bela, belatacept; BM, bone marrow; Chimp, chimpanzee; Cs, corticosteroids; CsA, cyclosporine; CVF, cobra venom factor; Cyno, cynomologus monkey; CyP, cyclophosphamide; Dac, Daclizumab; FK, tacrolimus; Gal abs, extracorporeal anti-Gal antibody adsorption; GTKO, 1,3-galactosyltransferase gene-knockout; hCD46, expression of the human regulator of complement, $\mathrm{hCD} 46$; hCD55, expression of the human regulator of complement, CD55; HT, H-transferase; LoCD2b, anti-CD2 monoclonal antibody; MMF, mycophenolate mofetil; Rhes, rhesus; Ritux, Rituximab; WBI, whole body irradiation; WT, wild-type. 


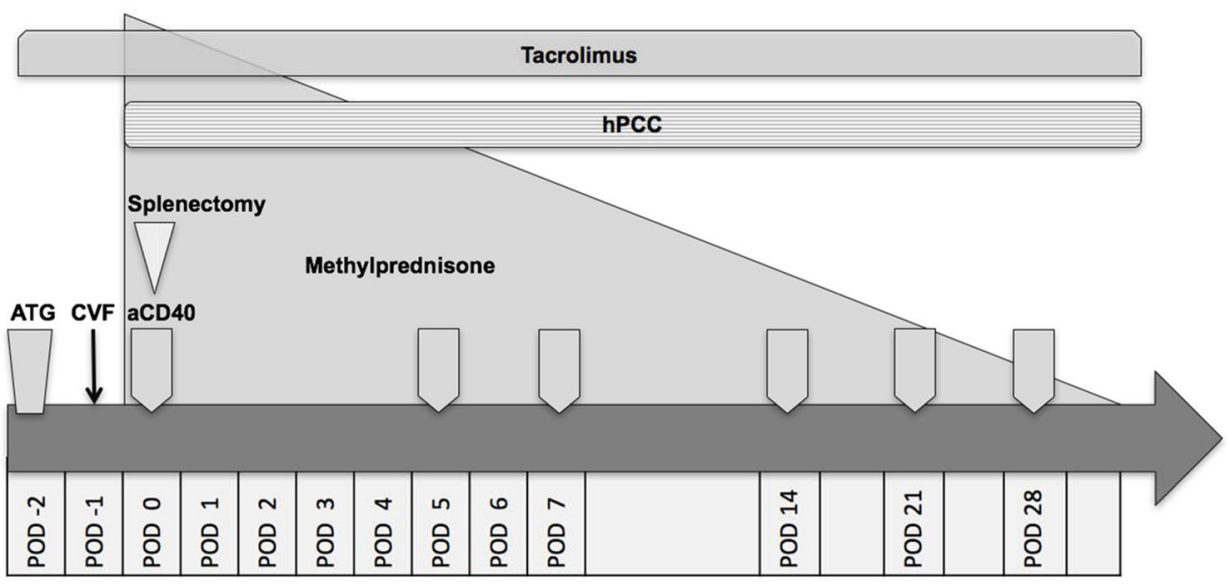

Figure 2.

Liver xenotransplant protocol for incorporating continuous exogenous administration of human coagulation factors and anti-CD40 mAb co-stimulation blockade resulting in nearly one month recipient survival.(10) aCD40, anti-CD40 mAb; ATG, anti-thymocyte globulin; CVF, cobra venom factor; hPCC, human prothrombin concentrate complex; POD, postoperative day. 